\title{
Overlapped Detection Via Approximate Entropy Estimation Against Flooding Attack in Mobile Sensor Networks*
}

\author{
Mihui Kim and Kijoon Chae \\ Department of Computer Science and Engineering, Ewha Womans University, Korea \\ mihui@ewhain.net, kjchae@ewha.ac.kr
}

\begin{abstract}
To achieve security in sensor networks, it is important to be able to defend against flooding attack recently considered as an extremely threatening attack. In this paper, we propose a flooding attack detection method as the first defense step, through approximate entropy estimation reflecting resource constraints of sensors. Each detector performs both basic estimation for its own region and overlapped estimation for its own and neighbor regions, against the mobility of attack node. Also, in order to enhance the accuracy of detection even in the various deployments of attack agents, we deploy hierarchically detectors according to network topology. This detector by entropy estimation is simplified by only multiplication calculation instead of logarithm, in addition to providing higher estimation precision of entropy compared to the conventional entropy estimation. Our simulation results indicate that this hierarchical defense is a feasible method, being especially promising for accurate decision through overlapped detection even in frequent handoffs of mobile attack agents.
\end{abstract}

\section{Introduction}

Wireless sensor networks (WSNs) are rapidly growing in their importance and relevance to both the research community and the public at large. Their promising applications are expected to help protect and monitor critical military, environmental, safety-critical, or domestic infrastructures and resources. To date, researches for security in sensor networks have laid disproportionate emphasis on basic security mechanisms, such as authentication or key management. Due to wireless characteristic or easy physical compromise of sensor nodes, these basic security services are indispensable. However, defense against possible attacks is also essential in order not to negate much of the promise sensor networks hold, because attacks can still be performed even if the network communication provides confidentiality and authenticity. Especially, threat against flooding attack, one of Denial of Service (DoS) attacks considered as the most threatening attacks in the wired network is not an exception even on sensor networks. In addition to, flooding attacks may permit real world damage to the health and safety of people, exceeding simple damages such as loss of sensory data or energy exhaustion of nodes. Although the operations of

This research was supported by the MIC, Korea, under the ITRC(Information Technology Research Center) support program supervised by the IITA. 
sensors are simplified due to their low cost and overhead, the vulnerabilities of each layer on sensors exist for different flooding attacks, like jamming, exhaustion, or flooding on transport layer. A.D. Wood et al. [1] presented the basic defenses like spread-spectrum or authentication against these attacks, but only these straightforward defenses are not sufficient for protecting the availabilities of sensor networks.

As one of defense mechanisms against attack on sensor networks, Serdar Sancak et al. [2] proposed two rough detection methods against spam attacks that generate dummy packets to deplete the energy of relaying nodes. Even though the second method using the frequencies of messages sent by the sensors in the same region is a little similar with our detection using entropy for distribution estimation of messages, a sink in [2] should count packets per each sensor, that is not scalable for many units, and they roughly mentioned that this detection method regarded a sensor node that generated $\delta$ times more packets as an anti-node. Anthony D.Wood et al. [3] designed a defense method against jamming attack on link layer by unwanted and disruptive radio propagation. Heuristically, it detects the attack if communication channel drops below a certain threshold, and then maps collaboratively a jammed region by modifying MAC, sending a brief, high-priority, un-acknowledge broadcast message. Fan Ye et al. [4] proposed SEF (Statistical En-route Filtering) in order to detect and drop false reports through validating by multiple keyed message codes (MACs).

Moreover, there has been an increasing interest to study and build the mobile nodes as well as static nodes in wireless sensor networks [5]. The mobile nodes in the network will enhance its capabilities, for example, they could be used to physically collect and transport data or to recharge and repair the static nodes in the network. However, in the view of security service, the mobility of nodes makes the solving problems more complicate and difficult. There are three types of mobility: node mobility, sink mobility, and event mobility [6]. First case is a general mobility of mobile sensor network, and is important to reorganize itself frequently enough to be able to function correctly. Second mobility is a special case of node mobility, for example, a human user requested information via a PDA while walking in an intelligent building. Last mobility is a case that the cause of events or objects to be tracked can be mobile. We assume the first general node mobility whether sensor nodes attached to livestock or human are mobile or nodes themselves can be mobile.

The structures of sensor networks are various according to their application, the flat structure by random deployment and the hierarchical structure centering nodes on a sink are representative. In this paper, we will explain our detection method based on the hierarchical sensor network consisting of sink (SINK), aggregate nodes (AN), and sensor nodes (SN). However, this detection might be easily applied to flat sensor network considered as a specific case of hierarchical network, one-layer network.

In this paper, we focus on flooding DoS attacks on mobile sensor environment. As the first proactive defense, we propose a flooding detection through practical entropy estimation. Entropy is a representative algorithm used for decision tree, one of the most popular classification algorithms in current use in data mining and machine learning, and has frequently used for differentiating the abnormal situation from the normal on the wired networks [7]. However, the original entropy calculation by logarithm could be a burden to limited ability of nodes. Thus, we pursue three goals for flooding attack detection on mobile sensor networks like following. 
- High detection accuracy and scalability through distributed detection with a few samples for various deployments of attack agents

- Detection possibility through overlapped detection even at frequent handoffs of mobile attack agents

- Low processing considering the limited ability of nodes

This paper is divided into five sections. In Section 2, we explain the basic of used approximate entropy method, as background. We introduce in Section 3 the proposed detection in detail. Next, we simulate our mechanism and explain our analysis of the simulation results, and a brief conclusion is finally presented.

\section{Approximate Entropy Estimator for a Discrete Random Variable}

To discriminate between attack and normal, we use the approximate entropy estimation by key information attached to all messages, based on our key mechanism [8]. In [8], the key management nodes calculate a key using key information provided by the holder of the key. Thus, we can imagine that if several compromised nodes would generate lots of DoS traffic, the distribution for used keys might be changed. If our key mechanism would not be used, this approximate entropy estimator could be used with the other information of message such as source identification, location information, or sensory data themselves for distinguishing unusual events. We pursued to simplify the entropy estimation considering limited capacity of nodes and result in accurate estimation, thus we used an approximate method for Bayesian entropy estimation [9].

Entropy estimation is one of statistical approaches used for distribution comparison where the measurements involved are discrete values. $M$-ary entropy function $H$ for a discrete random variable $\mathrm{X}$ which takes $M$ sorts of values $\left\{a_{1}, \ldots, a_{M}\right\}$ with probabilities $\left\{r_{l}, \ldots, r_{M}\right\}$ is $-\sum_{i=1}^{M} r_{i} \log _{2} r_{i}$. Thus, in conventional entropy estimation, occurrence probabilities $r$ of values $a$ are estimated by the maximal likelihood estimation method, i.e., $\check{r}=n / N$, in which values $a$ are assumed to be included respectively for $\mathrm{n} \equiv\left(n_{1}, \ldots, n_{M}\right)$ in the observed sample set of size $N$. However, unfortunately, the conventional entropy estimator is not optimum in the meaning of least square error [9].

Thus, we use basically the approximated Bayesian entropy estimator [9] as shown in equation (1), instead of the conventional estimator. In the equation (1), the previous work of [9] revealed that $s_{i}=0.5(i=1, \ldots, M)$, as center, and $K=20$ were sufficient from the viewpoint of approximation precision, and numerical experiments of [9] demonstrated that the proposed entropy estimation improved the estimation precision of entropy remarkably in comparison to conventional entropy estimation.

Equation (1) is seen a little complicatedly for our sensor network, but the only $n_{i}$ is changed in the entropy computation of each sample, and our simplified estimator will be explained in detail at next section.

$$
\hat{H}_{\text {Bayes }}=\sum_{i=1}^{M} s_{i}-\frac{1}{N+M} \sum_{i=1}^{M}\left(1+\log s_{i}\right)\left(n_{i}+1\right)-\sum_{k=2}^{K} \frac{1}{k(k-1)} \sum_{j=0}^{k}\left(\begin{array}{l}
k \\
j
\end{array}\right) \frac{(N+M-1) !}{(N+M+j-1) !} \cdot(-1)^{j} \sum_{i=1}^{M} s_{i}^{-j+1} \frac{\left(n_{i}+j\right) !}{n_{i} !}
$$




\section{Overlapped Detecting Approach}

\subsection{Basic Concept}

Our detection based on entropy estimation could use the source identification or location information if it is attached to message, sensory data that could be regularly changed as time, like temperature or light, or key information attached to message. We explain our detection based on our key management on hierarchical mobile sensor networks. However, this detection might be easily applied to flat sensor network considered as a specific case of hierarchical network, one-layer network.

The key idea of detection is that detector nodes such as SINK, aggregator, or cluster head, compute entropy on a sample of consecutive messages generated in each region. That is, at the previous work [8], sensor nodes or aggregators attach the information for a key used for authentication or encryption to messages, and the attached information consists of \{key space number, key information $\}$. Because every key is randomly distributed, the distribution for used key spaces would have a specific form normally. Thus, as a flooding attack detection method, we use entropy estimation on the key space number for a key used at messages. Respective entropy values of the normal case and the attack case that floods lots of messages, are very different [7].

For each detector node, simplifying equation (1), we result in equation (2) as our estimator for attack detection. $M$ can be the total number of key spaces in the key pool, or the subset number of key spaces. Value $\alpha$ and $\beta$ are constant by $N$ and $M$ that are fixed when this estimator is used $(\alpha=0.5 \cdot M, \beta=N+M-1)$. Also, $(-2)^{j}(j=1, \ldots, 20)$ is made to constants over temporary memory. Thus, the only $n_{i}$ changes in the entropy computation of each sample, and moreover any $n_{i}(i=1, \ldots, M)$ are not changed if we calculate the entropy through a sliding window of fixed width $\delta(<N)$ for consecutive messages as shown in figure 1. In conclusion, this estimator for flooding DoS detection is calculated with only multiplication operation.

$$
\tilde{H}_{\text {Bayes }}=\alpha-\sum_{k=2}^{20} \frac{1}{k(k-1)} \sum_{j=0}^{k}\left(\begin{array}{l}
k \\
j
\end{array}\right) \frac{\beta !}{(\beta+j) !} \cdot(-2)^{j} / 2 \sum_{i=1}^{M} \frac{\left(n_{i}+j\right) !}{n_{i} !}
$$

For speedy detection, detectors use $\delta$ sliding window for arrival packets. That is, each detection node updates each $n_{i}$ with the new arriving $\delta(1 \leq \delta \leq N)$ packets like figure 1 , recalculates $\tilde{H}_{\text {Bayes }}^{\prime}$, and compares it with the previous $\tilde{H}_{\text {Bayes }}$. If the gap between them is higher than a threshold, which could be chosen with the average and standard deviation of normally estimated entropy, the detection node notifies it to other detection node on higher layer (detection nodes toward a sink). If the sink judges the attack with estimation results of aggregators and itself, it performs followup measures in order to localize the damage as soon as possible. For the value of $\delta$, a trade-off between the speed of detection and the calculation overhead of entropy exists. Therefore, this value could be determined as the capacity of detection nodes. Also, for the value of $N$, the sample size for approximate entropy calculation, there is a trade-off between the accuracy of detection and the calculation overhead of entropy, 


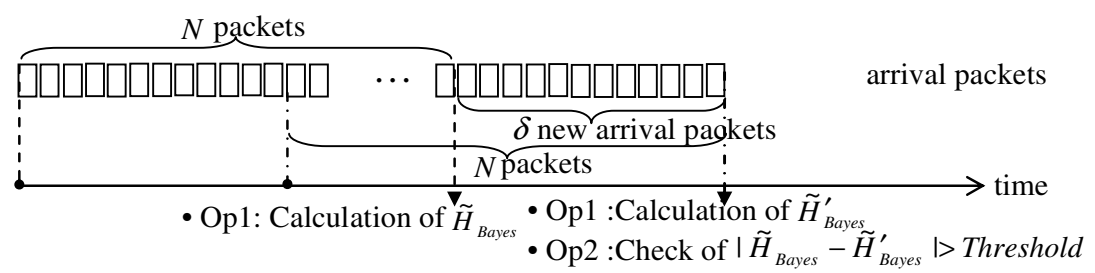

Fig. 1. Calculation and decision process on detection nodes

thus it also should be decided as the general generation speed of messages and the capacity of each detection node.

\subsection{Overlapped Detection on Hierarchical Mobile Sensor Network}

We assume that the subset of ANs and SINK perform two approximate entropy calculations for detection, a basic detection for its own management region and a overlapped detection for the region including a detection node's own region and its neighbors. Also, this subset of ANs could be changed as the control of SINK considering their residual energy and their covering region. Even if an AN does not perform the detection, the neighbor detection node can cover the region of the AN through overlapped detection. The reason why ANs perform the basic detection is to localize the damage by fast detection, and overlapped detection is for detecting moving attack agents. Also, SINK could synthesize the detection decision with estimation results of each region, aginst the various deployments of attack nodes.

Overlapped detection is that a detector calculates the approximate entropy for attack detection with the total traffic from sensor nodes of both its own region and neighbor regions, from where mobile sensor nodes could be moved to its own region. The goal of overlapped detection is to minimize the decrease of the detection performance by relatively reduction of attack traffic affecting a region though the mobility of attack node. The worst case could be that the handoff of an attack node would occurs frequently, thus it might be not disclosed by detectors. That is, the mobility of an attack node makes even the severe attack reduce to the stealthy attack of a region. If the mobility is considered, the centralized detection is better than the distributed method, but it is not scale to many sensor nodes.

Neighbor regions for overlapped detection are different according to network structure. For example, in the case of hexagonal grid-based network, there are 6 neighbor regions per a detector, and in the case of hierarchical sensor network, they are variable according to the partition of each layer. Neighbor regions of A3 in figure 2 are regions of SINK/A2/A4/A9/A10, and the information for neighbor regions on the same layer, such as A2/A4, could be conveyed as the combined information of higher detector, SINK, for overlapped detection, because the higher detector also periodically received the information of A2 and A4 regions.

We assume that the detectors are loosely time-synchronized, thus they periodically send their $n_{i}$ to detectors of neighbor regions through a high-priority message, and calculates the approximate entropy for the summation of $n_{i}$. If the detector does not receive $n_{i}$ from some regions, it requests the $n_{i}$ to them again. If the detector suspects the attack through overlapped detection process, it notifies the detection fact to 
neighbor detectors for the follow-up measure. Also, if the detector judges the attack through basic detection process, it sends its own $n_{i}$ to neighbor detectors for proactive detection against mobile attack agents.

\section{Evaluation}

To analyze the performance of our detection, we configured the simulation network and scenario like figure 2. This network consists of a SINK, 12 ANs covering respectively $50 \mathrm{SNs}$, and $650 \mathrm{SNs}$. The SINK has 5 key spaces, including especially a distributing key space 5 for newly deployed nodes, each AN at the first and second layer has the information for 3 and 2 key spaces respectively, and each SN has 3 randomly selected key sets coming under key spaces of higher AN. Even though the original key pre-distribution is performed randomly with the same probability for each key space, we intentionally distributes the key sets with probability $(0.1,0.2,0.3$, $0.1,0.3)$ for each key space. However, normally each SN generates the sensory data by the same probability with randomly selected key among its own key sets, thus the intended traffic generation probability for each key space is $(0.1,0.2,0.3,0.1,0.3)$.

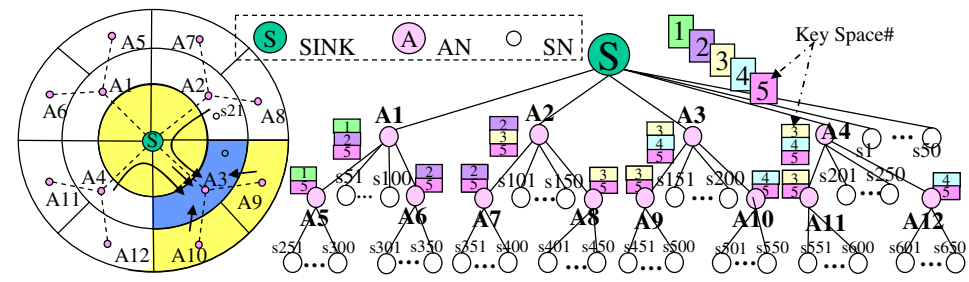

(a) A sample network for simulation

(b) Simplified model including SNs for (a)

Fig. 2. A sample network for simulation

\subsection{Detection Accuracy and Scalability}

At first, in order to estimate the possibility of our DoS detection based on approximate entropy, we intent to mount DoS attack during the specific period. As a result, we get a variation of estimated approximate entropy on SINK and true entropy by probability as shown in figure 3 . The $\mathrm{x}$-axis of this figure is estimate round as time goes on. For the calculation of approximate entropy $\tilde{H}_{\text {Bayes }}$, we use $s_{i}=0.5$ and $K=20$ as recommended in [9], $M=5$ due to 5 key spaces and $N=100$ or $N=500$. At each estimate round, the calculation of $\tilde{H}_{\text {Bayes }}$ is performed with the new 100 or 500 packets, that is $\delta=N$ as the worst case in the view of detection speed. During estimate period from 101 to 120 , we generate DoS attack traffic from SN s51, s52, s53 and s54 comprising $10 \%$ or $30 \%$ (Pattack in figure 3 ) of all packets. Thus, the true entropy by probability $(0.1,0.2,0.3,0.1,0.3)$ is 1.504788 as shown in figure 3 , and approximate entropy is also around the true entropy normally. But, during the simulated DoS attack period, the most approximate entropies increase considerably enough to easily detect the attack. Especially the bigger sample size $N$ and the severer attack exist, the more conspicuous entropy difference between normal and attack is. 
This estimation for DoS detection is distributed to ANs as well as SINK. Also, as the more nodes under the charge of a management node are, the more capacity the management node has, each $\mathrm{AN}$ as a detection node may not be a big burden to the detection load which is proportional to the received traffic from nodes under its charge. In comparison with [2] that a SINK should count packets per each sensor, we support scalability for many sensor nodes, because our SINK or higher layer ANs as a detection node deal with the only aggregated messages, and estimation is distributed.
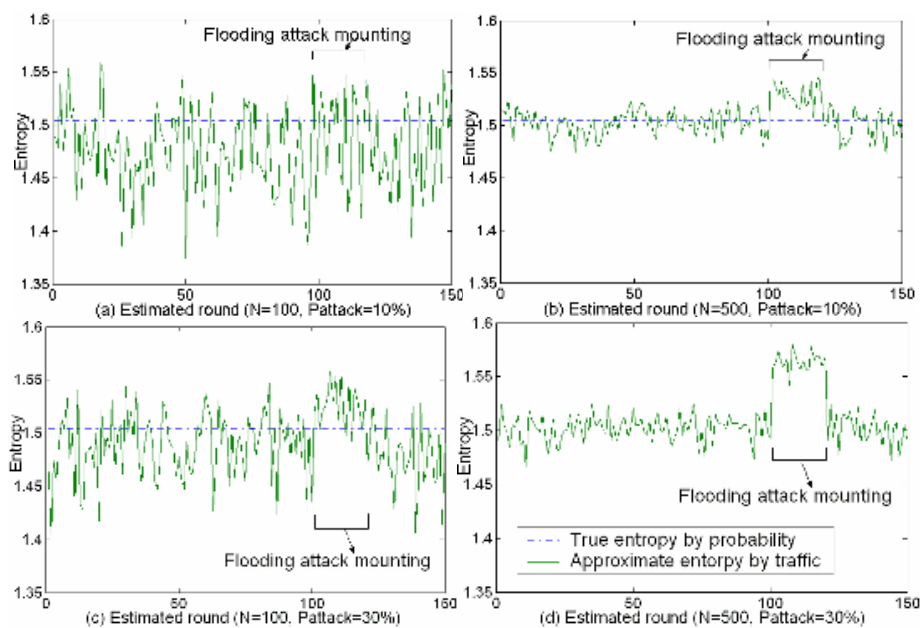

Fig. 3. Estimated approximate entropy and true entropy by probability

\subsection{Detection Possibility on Frequent Handoffs}

As the second simulation, we mount the DoS attack with 2 deployments of attack agents, concentrated attack and distributed attack. The attack agents in the former swarm around an $\mathrm{AN}$, that are $\mathrm{SN} \mathrm{s51,} \mathrm{s52,} \mathrm{s53,} \mathrm{and} \mathrm{s54} \mathrm{of} \mathrm{figure} \mathrm{2.} \mathrm{In} \mathrm{the} \mathrm{latter} \mathrm{case,}$ they are distributed to different ANs, which are SN s51, s101, s151, and s201. Like the first simulation, we mount a DoS attack during estimate period from 101 to 120 , and we estimate approximate entropy $\tilde{H}_{\text {Bayes }}$ for the DoS detection at SINK and AN1 respectively. Figure 4 shows the conspicuous increment of entropy at SINK in the concentrated attack case, but the entropy of AN1 is not changed nearly because the key spaces of 4 attack agents might cover all key spaces of AN1. On the other hand, in the distributed attack case of figure 4, the entropy increment at AN1 is more remarkable than at SINK because the attack traffic from only one attack agent makes the skew distribution of used key space number of AN1. Moreover, the uniformed distribution of attack agents in the view of SINK causes the less increment of entropy than their concentrated distribution. In conclusion, against various deployments of attack agents on hierarchical sensor networks, the synthetic decision needs for the accurate DoS detection. 


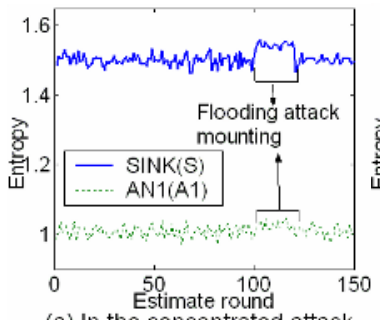

(a) In the concentrated attack

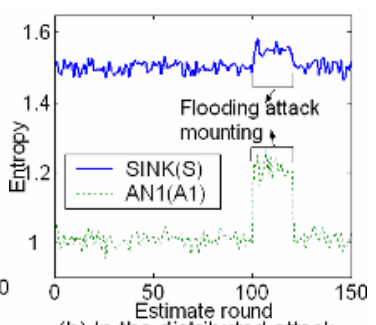

(b) In the distributed attack

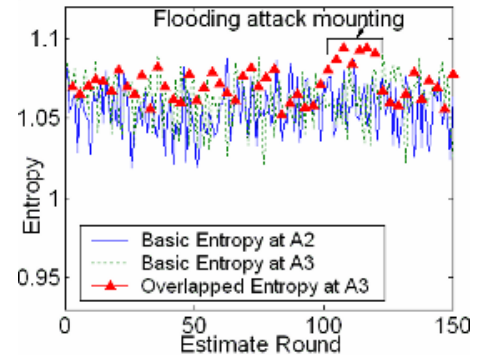

Fig. 4. Estimated entropy in two attack deployments

Fig. 5. Basic and overlapped entropy on $\mathrm{A} 2$ and $\mathrm{A} 3$ in attack of mobile nodes

As the last simulation result, figure 5 shows that basic and overlapped entropy in attack case of mobile agents. The mobile attack agents, from SN s101 to s104 mount stealthy attack during estimate period from 101 to 120, comprising 15\% attack traffic of all packets, and they wander over the regions of A2 and A3. In result, basic entropy on each region does not change due to less attack traffic by mobility, but overlapped entropy at A3 indicates the difference between normal and attack. Thus, the overlapped detection is important especially for the stealthy attack of mobile agents.

\subsection{Low Processing}

Our estimator is calculated with only multiplication, instead of logarithm operation of traditional entropy estimator. In the view of operation complexity, the multiplication is much simpler and needs less cycle on the CPU board than logarithm. It could diminish the burden for detection load to each detection node.

\section{Conclusion}

In this paper, we have suggested a detection method against flooding attack on mobile sensor networks using the approximate entropy estimation. This approximate entropy estimator is calculated with only multiplication operation instead of logarithm, thus it fits for nodes with limited resources. we localized the detection function to each subgroup of sensors as resources is permissible, and SINK synthesizes localized reports to detect the attacks even at various deployments of attack agents. Moreover, the detector estimates the overlapped entropy for mobile attack agents. We have also simulated our mechanism, in order to analyze the possibility of detection via the entropy estimation and the accuracy of detection even at handoffs of mobile attack agents. Finally, we obtained a prominent result even though the attack agents are concentrated or distributed, or they move.

\section{References}

1. Anthony Wood and John A. Stankovic, "Denial of Service in Sensor Networks," IEEE Computer, 35(10), pp.54-62, October 2002.

2. Serdar Sancak, Erdal Cayirci, Vedat Coskun and Albert Levi, "Sensor wars: Detecting and defending against spam attacks in wireless sensor networks,” ICC 2004 - IEEE International Conference on Communications, vol. 27, no. 1, pp.3668-3672, June 2004. 
3. Anthony D. Wood, John A. Stankovic and Sang H. Son, "Jammed-Area Mapping Service for Sensor Networks," $24^{\text {th }}$ IEEE International Real-Time Systems Symposium, December 2003.

4. Fan Ye, Haiyun Luo, Songwu Lu and Lixia Zhang, "Statistical En-route Filtering of Injected False Data," IEEE Infocom'04, March 2004.

5. Schmidt, S., Krahn, H., Fischer, S., Watjen, D., "A Security Architecture for Mobile Wireless Sensor Networks,” ESAS'04, LNCS3313 (2005), pp.166-177.

6. Karl, H., Wilig, A. "Protocols and Architectures for Wireless Sensor Networks," John Wiley \& Sons, Ltd., 2005, pp.62-63.

7. Laura Feinstein, et al., "Statistical Approaches to DDoS AttackDetection and Response," Proc. of the DARPA Information Survivability Conference and Exposition, 2003.

8. Mihui Kim and Kijoon Chae, "A Hierarchical Key Management based on Multiple Regression in Mobile Sensor Networks," Submitted to a Conference.

9. Yasunari Yokota, "An Approximate Method for Bayesian Entropy Estimation for a Discrete Random Variable," Proc. of the $26^{\text {th }}$ IEEE EMBS, September 2004. 